Tohoku J. exp. Med., 1983, 141, Suppl., 29-39

\title{
Standardization of Methods in the Epidemiology of Diabetes Mellitus
}

\author{
Peter H. Bennett \\ Epidemiology and Field Studies Branch, National Institute \\ of Arthritis, Diabetes, Digestive and Kidney Diseases, \\ Phoenix, Arizona, USA*
}

At the Asia and Oceania Congress on Endocrinology held in Singapore in 1978 a plea was made for the adoption of standardized methods for glucose tolerance testing and other methodologic aspects of diabetes epidemiology. Since that time considerable progress has been made. Further discussions of the desirability of standardizing epidemiologic methods in diabetes also took place later in 1978 at an international conference on the Epidemiology of Diabetes and its Macrovascular Complications ${ }^{1)}$. Some of the focus was on glucose tolerance testing, but there was also discussion of standardized methods for micro- and macrovascular complications of diabetes. In 1979, the National Diabetes Data Group (NDDG) in the United States reported its recommendations on standardization $^{2)}$, and in 1980, the World Health Organization published the report of its Expert Committee $^{3)}$, which detailed the new classification of diabetes mellitus and proposed standardized diagnostic criteria, which were based in large part on those of the NDDG. The present paper reviews some of the reasons for adopting standardized methodologies and sets forth some further proposals.

\section{General Considerations}

The need for standardization

Standardized methods of assessment are needed for any research study in order to communicate the results, and to permit others to judge the validity of the conclusions. The need becomes particularly relevant when a study involves different observers, or when a comparative study is conducted in different populations, or in the same population at two different points in time. If standard mehtods are not adopted there is little reason to believe that similarities or differences between populations can not be accounted for by methodologic differences.

Desirable characteristics of methods for epidemiologic studies

There are cartain characteristics of methods which are necessary, or desirable,

* 1550 E. Indian School Road, Phoenix, Arizona, USA 85014 
if wide spread use is to be recommended. Foremost, the method recommended must be valid. That is it should measure what it is purported to do. For population studies, the method should have a relatively high degree of specificity. That is a negative test should have high likelihood of indicating normality with regard to that characteristic in question. A high degree of sensitivity-the ability of the method to detect a true positive-is generally not so critical.

Variability is associated with all measurements, and relatively high variability, i.e. poor reproducibility, may cause considerable problems in relation to individual classification but may be more tolerable for group comparisons, since its main undesirable effect is to reduce the likelihood that a true difference will be found between groups when differences, in fact, exist. On the other hand a statistically significant difference between groups cannot be attributed to differences in the variability of the measure used to detect the difference. Variability is determined by measuring the reproducibility of a measurement.

In order to be applied by different investigators working in different populations epidemiologic methods should be as simple as possible. A simple method applied to a large group of subjects, even though it may have lower sensitivity and reproducibility may be more powerful than a more complicated method which, for logistic reasons, can only be applied to small numbers of subjects.

Wherever possible epidemiologic methods should be quantitative since this increases the ability to perform comparisons, and quantitation is usually associated with some increase in the degree of objectivity of the measurement. Best of all, if a method can give a "hard" output, such as an electrocardiogram or a retinal photograph, the ability to make comparisons is much enhanced since data from two or more populations can be examined simultaneously by the same team of observers, or determined in the same laboratory. This minimizes the effects of observer bias, which are difficult to control whenever a subjective judgement is made.

\section{Standardization of historical data}

Acquitision of historical data is best done by asking standardized questions. The questions are best posed in the form of a self-administered questionnarie, or by trained interviewers. These techniques eliminate or minimize interviewer bias, whereas an unstructured medical history may be subject to extreme bias. Although not always feasible, data, normally obtained by history, can be sometimes retrieved and validated from other records and when possible this is to be preferred since it eliminates reliance on the memory of the subject.

Some standardized medical history questionnaires suitable for use in diabetes investigations which have been widely tested are already available e.g., the Rose questionnaire for coronary heart disease ${ }^{4}$, WHO study of vascular disease and diabetes questionnaire ${ }^{5)}$. 


\section{Physical examination in epidemiologic studies}

In epidemiologic studies the physical examination should be totally systematic, limited to a few items of special interest, and, wherever possible, should be performed without knowledge of the patient's clinical history or laboratory findings. Simple examination techniques are to be encouraged and the reproducibility of these techniques in the hands of those making the observations should be tested and documented. Surprisingly large variability is frequently encountered, and its measurement may itself prove to be a very valuable training experience for the observers. Furthermore, physical examination findings should be recorded according to a predetermined set of rules, and are best recorded on a precoded form, where all the possible options for recording are specified. Attempting to code physical examination findings from an unstructured examination, can be fraught with error, and is often impossible. Attempts should be made to minimize clinical judgement. Physical examination data can often be acquired more systematically, reliably and reproducibly by nonphysician personnel, who have been trained to record a limited set of findings. Such techniques, and the recording of observations in a quantitative fashion, help to minimize observer bias.

\section{Standardization of laboratory methods}

Wherever studies are to be done in different locations or using different teams of data gatherers, it is important to standardize the method of collection and processing of the data. This applies to biochemical determinations equally to data such as electrocardiographic or radiographic information. The easiest and simplest way to minimize variation is to use a central facility, when possible. For example, if blood can be collected appropriately, and then sent to a central laboratory from different field sources, standardization of many potential variables can be achieved. In general, quality control measures are less complicated if determinations are made in a single laboratory, and the precision and reproducibility of the assay can be maintained given adequate standardizaiton of equipment (or observers). Control and duplicate determinations can be made systematically at frequent intervals. The use of two or more laboratories demands more stringent standardization and quality control, as the variability will almost always be higher than when a single central laboratory is utilized.

\section{Reporting of results}

Comparisons of findings made in different populations are often desired. This is possible provided that simmilar methods have been used and usually involves a reanalysis of existing data. If this is done after the fact the term "pooling" is often applied to the joint analysis of findings. The need for "pooling" can be reduced, and meaningful comparisons can be made from different 
studies if standardized methods of reporting are adopted. For example, it is now possible to report the frequency of well defined categories of diabetes, since both standardized diagnostic criteria and a classification scheme have been adopted. Furthermore, the results of epidemiological studies should be reported according to standardized age groups, which are internationally recognized, and which minimize the digit preference, which often occurs with stated age. The use of standardized groupings for such characteristics as age obesity, etc., facilitates comparisons with studies conducted elsewhere. This need not inhibit the reporting of alternative groups, but should be done in addition to, rather than in place of, the standardized intervals.

In many studies insufficient time and effort is expended in the planning phases. Especially in collaborative studies, planning should include exhaustive discussion of the goals of the study, and the specific comparisons which will be made. It is then possible to identify the specific items of data which will be ultimately required to make these comparisons. Once this is achieved, attention should be turned to the standardized definition and acquisition of these data, and of methods to ensure that the comparability of data obtained by different observers can be documented.

Once this is achieved a pilot project is useful to ascertain if the methods are standardized, and are sufficiently reproducible between centers and/or observers so that the data gathered will be valid for testing the hypotheses proposed, and for making desired comparisons.

\section{Specific Methods for Use in Diabetes Epidemiology}

\section{Diabetes and glucose intolerance}

The proposals made by the WHO Expert Committee and their wide spread adoption by workers in many parts of the world represents a major step forward in diabetes epidemiology ${ }^{3)}$. The classification scheme proposed enables categorization of individuals according to standardized definitions and should do much to enhance the certainty with which conclusions can be made concerning variation in the prevalence and incidence of diabetes in populations, as well as in diabetes research in general. Agreement on diagnostic criteria and upon the oral carbohydrate (glucose) load to be used for testing will enable a much more standardized basis for the reporting of results from population studies.

A few minor problems remain with the classification and should be resolved. For studies of insulin dependent diabetes mellitus (IDDM), the definition of insulin dependency should be tightened, particularly in terms of minimum criteria for what constitutes ketosis-proneness. Since the present definition is open to some judgement, biases may appear in the categorization of individual subjects. Another problem in the WHO diagnostic criteria concerns the rounding errors from $\mathrm{mg} / \mathrm{l}$ to $\mathrm{mmol} / \mathrm{l}$. This is a problem particularly with the fasting values, where a concentration of $7 \mathrm{mmol} / 1$ is equal to $126 \mathrm{mg} / 100 \mathrm{ml}$, and not $120 \mathrm{mg} / 100 \mathrm{ml}$ as 
stated in the report; a value of $8 \mathrm{mmol} / \mathrm{l}$ is equivalent to $144 \mathrm{mg} / 100 \mathrm{ml}$, and not $140 \mathrm{mg} / 100 \mathrm{ml}$; and $11 \mathrm{mmol} / \mathrm{l}$ is equivalent to $198 \mathrm{mg} / 100 \mathrm{ml}$ and not $200 \mathrm{mg} /$ $100 \mathrm{ml}$. While these discrepancies probably make no difference to the clinical management of individual subjects, a difference of $4 \mathrm{mg} / 100 \mathrm{ml}$ at the tail of a distribution curve, such as is the case with fasting glucose especially, can result in an appreciable difference in the percentage of subjects who are classified as abnormal in group data. This problem may also be aggravated by rounding errors and digit preference, which are apt to occur in the laboratory. The simplest solution would be to either work in $\mathrm{mg} / \mathrm{dl}$ or to provide more accurate conversions, i.e., $\geqq 140 \mathrm{mg} / 100 \mathrm{ml}$ equals $\geqq 7.78 \mathrm{mmol} / 1 ; \geqq 120 \mathrm{mg} / 100 \mathrm{ml}$ equals $\geqq 6.67 \mathrm{mmol} / 1 ; \geqq 200 \mathrm{mg} / 100 \mathrm{ml}$ equals $\geqq 11.1 \mathrm{mmol} / 1$.

For epidemiological purposes only the National Diabetes Data Group criteria suggested that a $2 \mathrm{hr}$ postload plasma glucose value was sufficient for classification. This question is not addressed in the WHO diagnostic criteria, but virtually all subjects who have a fasting glucose level in excess of $140 \mathrm{mg} / 100 \mathrm{ml}$ also have a glucose level in excess of $200 \mathrm{mg} / 100 \mathrm{ml}$, and usually in excess of 250 $\mathrm{mg} / 100 \mathrm{ml}$, following an oral glucose load. The suggestion that for epidemiologic purposes a single glucose determination two hours after an oral glucose load is sufficient to categorize the subjects, therefore, seems reasonable and in many instances would simplify field procedures considerably.

\section{Assessment of Microangiopathy}

\section{Retinopathy}

There are two widely used methods available to assess retinopathy-opthalmoscopy and retinal photography. Careful opthalmoscopy following mydriasis used to be considered the method for choice for the detection of retinal lesions ${ }^{6}$. Even with adequate mydriasis, however, the retina may be inadequately visualized because of the presence of corneal or lens opacities, or a cloudy vitreous. It is important to record the presence of these abnormalities, since they may be more common in the diabetic, and the frequency of retinopathy in persons where an inadequate view of the vitreous chamber and retina has been obtained and must be known.

A suggested scheme for recording retinopathy is as follows: The changes found in each eye should be separately recorded.

For each eye, record:

Visualization of Fundus

Right Left

Adequate-1 Inadequate- 0

For (a), (b) code the number of lesions seen $-0=$ none, $1=$ one, $2=2-5,3=6$ or more, $9=$ not visualized

Right Left

(a) Red dots: 
Microaneurysms or small intraretinal hemorrhages

(b) White dots:

Hard exudates

Soft exudates

Drusen

For (c) and (d), code $0=$ no/yes $=1$

(c) Proliferative changes:

(i) Neovascularization

(ii) Vitreous hemorrhage

(iii) Retinal detachment (non-traumatic)

(iv) Fibrous strands

(d) Photocoagulation scars

(For treatment of diabetic retinopathy)

If the individual elements of retinopathy are recorded it is possible to examine the occurrence of each with various degrees of glucose intolerance and with the diagnosis of diabetes and impaired glucose tolerance.

The presence of "red dots" (a) in the absence of proliferative changes is the essential basis for the diagnosis of "background retinopathy."

The differentiation of hard exudates and drusen may prove difficult; nevertheless, the examiner should attempt to make the distinction. However, since "white dots" are not specific to diabetes, it is suggested that the presence of only "white dots" alone should not be a criterion for background retinopathy.

Since proliferative changes $(c \& d)$ represest the more advanced stage of retinopathy and often coexist with background retinopathy, it suggested that if both types are recorded the subject should be reported as having as "proliferative retinopathy."

Considerable advances in the techniques available for retinal photography have now made it possible to obtain high quality retinal photographs even without mydriasis. This has been possible by the application of infrared viewing capability combined with a cathode ray monitor to enable the retina to be focused and positioned for the photograph. Advances in optics permit the use of a $45^{\circ}$ Fields, which allows much larger area of the ratina to be visualized in a single photograph. Indeed our own experience with such an instrument $\dagger$ has shown that we are able to detect red lesions (microaneurysms) approximately twice as often using such an instrument, as with the conventional $30^{\circ}$ fundus camera. The wider angle of the $45^{\circ}$ camera permits in a single photograph visualization of the equivalent of two-three fields of the $30^{\circ}$ instrument. Thus, the $45^{\circ}$ camera enlarges the area of retina which can be evaluated by taking a small number of

† Canon ${ }^{\circledR}$ non-mydriatic retinal camera CR2-45 NMA. 
photographs. Furthermore, the sensitivity of this photographic method of detection appears to be as great as we obtain with ophthalmoscopy.

A further advantage of the infrared focusing is that, in addition to not requiring mydriasis (although a short acting mydriatic is still useful, the procedure of retinal photography much more acceptable to the patients, as well as much easier for the camera operator. In our own experience satisfactory retinal photographs are obtained approximately twice as frequently with such an instrument as with the older $30^{\circ}$ camera with a light focusing device.

Retinal photographs provide an objective and portable record, which may be evaluated independently and without knowledge of the patient's clinical status. Photography, however, cannot entirely replace ophthalmoscopy, since the latter is necessary to recognize vitreous hemorrhage, which may obscure the retina. However, a combination of ophthalmoscopy and retinal photography provides a sensitive and specific measure of the presence of diabetic retinopathy.

It seems likely that for epidemiologic purposes two views of each retina, one with the optic disk at the base of the photograph, and the other with the optic disk at the edge and including the macula in the center of the photograph, represent adequate views for the epidemiological diagnosis of retinopathy.

Since retinal photographs are capable of recording small lesions, photographs should be read by two observers for the presence of "red dots", "white dots" (exudates and drusen), and proliferative changes, or evidence of photocoagulation. Any disagreements should be resolved by a third reader.

For reporting purposes, the results of retinal photography can be summarized in the same manner as recommended for ophthalmoscopic findings.

\section{Renal disease}

Proteinuria is a sensitive index of renal disease in diabetes and can be measured systematically in epidemiologic studies. Although proteinuria lacks specificity for the diagnosis of diabetic renal disease, its detection in groups of cases and controls can give useful information on attributable frequencies. "Clinical proteinuria" has been traditionally detected by use of commercially available bromophenol test strips. Such strips have a sensitivity of approximately $20 \mathrm{mg} /$ $100 \mathrm{ml}$, but, because of the wide variation in urine concentration, this equates to a detection limit for protein excretion of $100-500 \mathrm{mg} /$ day. Quantitative urine protein measurements on "strip positive" urines, and the expression of these concentrations per gram of unine creatinine have been recommended for epidemiologic purposes in the past ${ }^{6)}$. However, reliable methods of measuring protein in much lesser amounts are available, and it is now possible using either nephelometric techniques using an antiserum to total human serum protein, or albumin ${ }^{7)}$, or using radioimmunoassay with an albumin specific antibody ${ }^{8)}$, to detect and quantify proteinuria in the truly physiologic range.

As collection of $24 \mathrm{hr}$ unine samples is impossible in large scale epidemiologic 
studies, such methods, can be usefully combined with urine creatinine measurement, and calculation of a urine protein, or albumin/ creatinine ratio in all subjects.

In view of current interest in the predictive value of microproteinuria for the development of renal disease ${ }^{9,10)}$, such methods can now be recommended for wide spread application. If the goal is only to report on the frequency of "clinical proteinuria", then a screening procedure with a bromophenol strip, followed by quantitative measurement of the protein, preferably using the nephelometric or radioimmunoassay procedure, and reporting urine protein/creatinine ratios $\geqq 0.5$ will provide a standardized method of documenting the frequency of "clinical proteinuria". Subjects who screen negative by the bromophenol strip can reasonably be assumed to be excreting less than $0.5 \mathrm{~g} /$ day, and therefore, have a urinary protain/creatinine ratio of $<0.5$.

\section{Renal failure}

Measurement of the serum creatinine level provides a stable and reliable index of end stage renal failure provided good quality control is maintained in the laboratory. The serum creatinine level is reliable and reproducible within subjects on a day to day basis. There are, however, systematic differences between normal serum creatinine levels in men and in women, and a fall to approximately $50 \%$ in creatinine clearance must occur before elevations of the serum creatinine concentration are seen. For epidemiologic purposes reporting the frequencies of serum creatinine levels of $\geqq 1.50 \mathrm{mg} / 100 \mathrm{ml}$ in men and $\geqq 1.30 \mathrm{mg} / 100 \mathrm{ml}$ in women provides a standardized method of documentation of end stage renal failure.

\section{Assessment of Macroangiopathy}

Standardized methods for the documentation of macroangiopathy have been developed largely by cardiovascular disease epidemiologists ${ }^{11}$. Unfortunately, diabetes epidemiologists often perform poorly when standardizing methods for the recognition of vascular disease, and conversely, cardiovascular disease epidemiologists often fail to document diabetes and glucose intolerance effectively. It is recommended that for studies of diabetes epidemiology the same standardized methods developed by the cardiovascular epidemiologists be utilized, whenever possible $^{12)}$.

\section{Hypertension}

Blood pressure is a very variable measurement, but nevertheless an important one. In epidemiologic studies blood pressure should be measured with a mercury sphygmomanometer with a sufficiently large cuff applied correctly to the arm. Under optimal circumstances a random zero sphygmomanometer should be used to minimize digit preference, and in any event both systolic and diastolic blood 
pressure should be measured to the closest $2 \mathrm{~mm}$ of mercury. It is important to define precisely the end point to be used for the diastolic measurement, and to increase comparibility with other investigations it may be necessary to record both phase IV and V diastolic pressures. A variety of training films and methods are available to standardize observers and which serve to minimize observer bias and increase reproducibility ${ }^{12}$.

\section{Coronary heart disease}

The "Rose Questionnaire" has been widely used as a method for obtaining a history of coronary heart disease ${ }^{4)}$. However, in populations where the frequency of coronary heart disease is low, the questionnaire performs relatively poorly because it has low sensitivity and questionable specificity. A much both method in my view is to obtain a standard twelve lead electrocardiogram. When the methods for obtaining the electrocardiogram are standardized, and the electrocardiogram is read by two or more trained observers by the Minnesota Code, an epidemiologically proven method for the recognition of probable myocardial infarction and possible coronary heart disease is available ${ }^{11}$. This still has low sensitivity, but does have high specificity and is objective and has good reproducibility.

For reporting purposes, it is suggested that diabetes epidemiologists follow the system used by the WHO Multinational Vascular Disease Study in Diabetics ${ }^{13)}$ coding the outcome as follows: Probable MI, codes 1.1, 1.2, or 7.1; and Possible CHD 1.3, 4.1, 4.2, 4.3 and 5.1, 5.2, 5.3. The performance of the Minnesota Code has been widely evaluated in epidemiologic studies of heart disease.

\section{Peripheral vascular disease}

While of great interest to the epidemiologist, objective methods of documenting peripheral vascular disease have not been widely used and developed for epidemiological purposes. A questionnaire for intermittent claudication has been developed ${ }^{4)}$ and has been used in some diabetes studies, e.g., WHO Multinational Study ${ }^{5}$. While a crude measure, amputation in the absence of a clear-cut history of trauma provides the hardest end point available for lower limb peripheral vascular disease. However, this is an insensitive index of peripheral vascular disease, but nevertheless is highly spicific and, therefore, useful for epidemiologic purposes provided sufficiently large samples are available.

A more sensitive measurement of peripheral vascular disease, but of quite different significance, is the detection of vascular calcification in the lower limb vessels. Radiographs of the feet, a lateral view of the calf, and an anteroposterior view of the thigh, taken using soft tissue techniques, provide evidence of vascular calcification of the media and intima of the arteries. These radiographs can be evaluated objectively, and compared by reading radiographs from different groups of subjects simultaneously. No internationally recognized standards for 
interpretation and reporting have yet been proposed.

The documentation of cerebral vascular disease is also difficult. However, some attempts to devise standardized methods for obtaining a history of transient ischemic attack, stroke, etc., have been developed ${ }^{11)}$, but have not been widely applied in epidemiologic studies of diabetes, and therefore, cannot be generally recommended at this time.

Other noninvasive techniques for the detection of peripheral disease, such oscillometry and Doppler measurements, are available, but none have been shown to be of value in large scale studies, and no specific recommendations can be made at this point.

All of the above methods for the measurement of peripheral vascular disease require further methodologic research prior to their general adoption as standard methods in the epidemiology of diabetes.

\section{Summary and Conclusions}

While considerable advances have been made in devising standardized methods for epidemiologic studies, it is only recently the standardized methods for use in diabetes epidemiology have been seriously considered, It is hoped that the present paper will provoke further attention to these matters and result in further discusions of areas where it appears possible to reach a concensus. Research into the performance of certain of the methods mentioned is urgently needed, and it is hoped that epidemiologists interested in diabetes will take up this challenge to prove or disaprove that some of the recommendations and suggestions made in this paper are useful and worthwhile.

\section{References}

1) Proceedings of the Kroc Foundation International Conference on Epidemiology of Diabetes and its Macrovascular Complications (1979) Diabetes Care, 2, 63-226.

2) National Diabetes Data Group (1979) Classification and diagnosis of diabetes mellitus and other categories of glucose intolerance. Diabetes, 28, 1039-1057.

3) WHO Expert Committee on Diabetes Mellitus Second Report (1980) Technical Report Series 646, World Health Organization, Geneva, pp. 80.

4) Rose, G., McCartney, P. \& Reid, D.D. (1976) Self-administration of a questionnaire on chest pain and intermittent claudication. Brit. J. Prev. Soc. Med., 31, 42-48.

5) Jarrett, R.J., Keen, H. \& Grabauskas, V. (1979) The WHO multinational study of vascular disease in diabetes. 1. General description. Diabetes Care, 2, 175-186.

6) Bennett, P.H. (1979) Recommendations on the standardization of methods and reporting of tests for diabetes and its microvascular complications in epidemiologic studies. Diabetes Care, 2, 98-104.

7) Killingsworth, L.M., Britain, C.E. \& Woodard, L.L. (1975) Automated immunochemical method for determination of urinary protein of plasma origin. Clin. Chem, 21, 1465-1468.

8) Keen, H. \& Chlouverakis, C. (1963) An immunoassay for urinary albumin at low concentrations. Lancet, 2, 913-914.

9) Aronoff, S.L., Schnider, S., Smeltzer, J., Mackay, W., Tchou, P., Rushforth, N.B., Miller, M. \& Bennett, P.H. (1981) Urinary excretion and renal clearance of specific 
plasma proteins in diabetes of short and long duration. Diabetes, 30, 656-663.

10) Viberti, G.C., Jarrett, R.J., Mahmud, V., Hill, R.D., Argyropoulos, A. \& Keen, H. (1982) Microalbuminuria as a predictor of clinical nephropathy in insulin-dependent diabetes mellitus. Lancet, 1, 1430-1432.

11) Rose, G. \& Blackburn, H. (1968) Cardiovascular survey methods. WHO Monograph Series No. 56, WHO, Geneva.

12) Rose, G., Keen, H. \& Jarrett, J. (1979) Epidemiologic methods in diabetic macrovascular disease. Diabetes Care, 2, 91-97.

13) WHO Multinational Study Group. The prevalence of macro- and microvascular disease in diabetes. In preparation. 